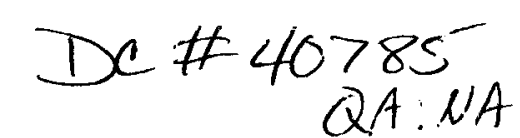

MOL . 20040629.0361

Technical note: 3210 Modeling; 3230 Numerical solutions; 5104 Fracture and flow; 1832

Groundwater transport; 1875 Unsaturated zone.

\title{
Improved Scheme for Modeling Mass Transfer between Fracture and Matrix Continua with Particle Tracking Method
}

Lehua Pan, Yongkoo Seol and Gudmundur S. Bodvarsson

\author{
Earth Sciences Division \\ Lawrence Berkeley National Laboratory \\ Mail Stop: 90-1116, One Cyclotron Road, Berkeley, CA 94720 \\ LPAN@LBL.GOV
}

\begin{abstract}
The dual-continuum random-walk particle tracking approach is an attractive simulation method for simulating transport in a fractured porous medium. In order to be truly successful for such a model, however, the key issue is to properly simulate the mass transfer between the fracture and matrix continua. In a recent paper, Pan and Bodvarsson (2002) proposed an improved scheme for simulating fracture-matrix mass transfer, by introducing the concept of activity range into the calculation of fracture-matrix particle-transfer probability. By comparing with analytical solutions, they showed that their scheme successfully captured the transient diffusion depth into the matrix without any additional subgrid (matrix) cells. This technical note presents an expansion of their scheme to cases in which significant water flow through the fracture-matrix interface exists. The dual-continuum particle tracker with this new scheme was found to be as accurate as a numerical model using a more detailed grid. The improved scheme can be readily incorporated into the existing particle-tracking code, while still maintaining the advantage of needing no additional matrix cells to capture transient features of particle penetration into the matrix.
\end{abstract}




\section{Introduction}

Modeling mass transfer through the fracture-matrix interface is one of the critical issues in simulating of transport in a fractured porous medium. Within the framework of a dualcontinuum, random-walk, particle-tracking approach, mass transfer through the fracture-matrix interface is simulated by a random-switch process (Pan et al. 2001). This random-switch process is controlled by the fracture-matrix particle-transfer probability at which a particle will enter the other continuum after a given time duration (time step). As a result, the issue of modeling mass transfer through the fracture-matrix interface in the dual-continuum particle-tracking model becomes one of how to determine the fracture-matrix particle-transfer probability. In calculating fracture-matrix particle-transfer probability, two critical parameters (i.e., the characteristic distance and the matrix volume) are usually assumed to be constant, as in other dual-continuum numerical methods (Pan et al., 2001). Such approximations, however, could lead to significant simulation errors, especially in the case of media with larger fracture spacing, because physically particles only have limited penetration depth into the matrix after they are injected into the fractures, and such a depth is inherently transient. A multiple interactive continuum (MINC) approach has been proposed in the past to capture this transient feature of penetration depth under the framework of the finite-difference methods (Pruess and Narasimhan, 1985). The basic idea of MINC approach is to use multiple grid cells to represent the matrix corresponding to each fracture cell (Figure 1). The disadvantage of this approach is that with $\mathrm{MINC}$, the required computation resources for any given problem often are much larger than using the dualcontinuum approach, which is a serious limitation for many real-world applications. 


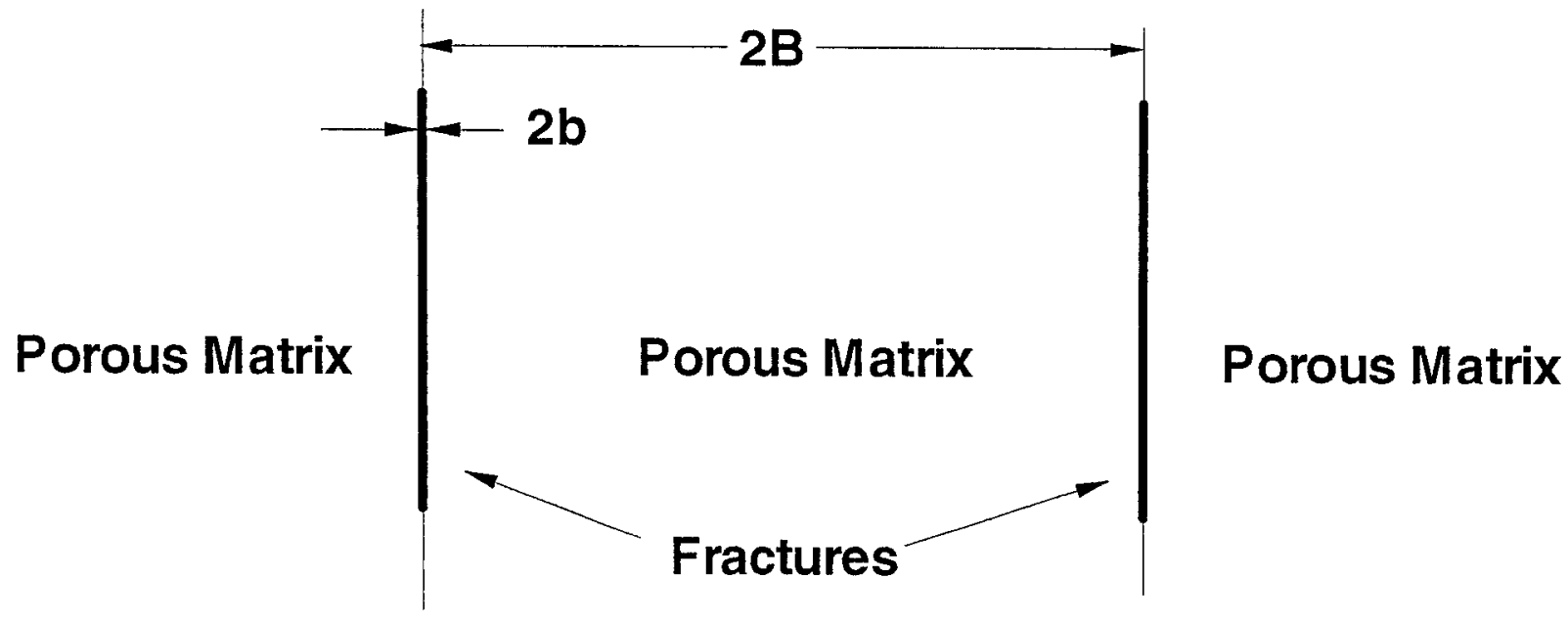

Dual continuum approach

MINC approach

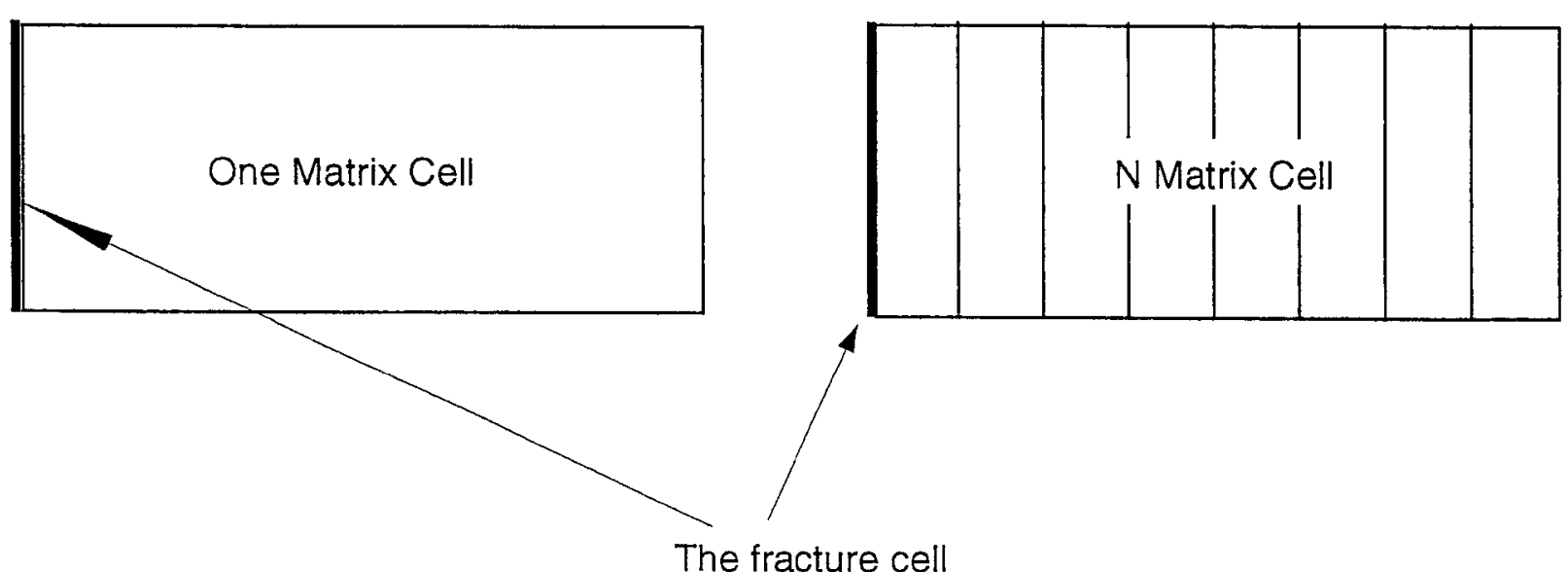

Figure 1. Schematic of a fracture-matrix system (upper portion) and its grid representations in a dual-continuum model and a MINC model (lower portion), respectively. 
In a recent paper, Pan and Bodvarsson (2002) found a way to capture the transient feature of penetration depth without using additional matrix grid cells (thus maintaining optimum efficiency). Instead of using additional matrix grid cells, they proposed to use a transient activity range of particles to mimic the transient penetration depth, and to connect the particle transfer probability with the activity range by defining two key parameters, the characteristic distance $\mathrm{S}_{\mathrm{fm}}\left(\mathrm{t}_{\mathrm{p}}\right)$ and the matrix volume $\mathrm{V}_{\mathrm{m}}\left(\mathrm{t}_{\mathrm{p}}\right)$, in the following manner:

$$
S_{\mathrm{fm}}\left(t_{\mathrm{p}}\right)=S_{\mathrm{frn}} \frac{B^{\prime \prime}\left(t_{\mathrm{p}}\right)}{B}
$$

$$
V_{m}\left(t_{p}\right)=V_{m} \frac{B^{\prime \prime}\left(t_{p}\right)}{B}
$$

where $B^{*}\left(t_{p}\right)$ is the activity range, the value of which varies from 0 to $B$, the maximum activity range (e.g., one-half the fracture spacing for a system of parallel-plate fractures separated by porous rock). $S_{\mathrm{fm}}$ and $\mathrm{V}_{\mathrm{m}}$ are the maximum characteristic distance and the maximum matrix volume for a given fractured porous rock, respectively. The age of a particle $\left(t_{\mathrm{p}}\right)$, defined as the time elapsed since injected into the medium, can be easily tracked by particle tracking methods (a unique advantage that is not available in finite-difference or finite-element numerical methods). The key to success for the above idea, as found by Pan and Bodvarsson (2002), is that the function $\mathrm{B}^{*}\left(\mathrm{t}_{\mathrm{p}}\right)$ can be derived based on the analytical solution of the one-dimensional diffusion process along the fracture-matrix dimension (the $4^{\text {th }}$ dimension in dual-continuum model). The final formula for calculating the activity range was defined as follows (see their original paper for detailed derivations): 


$$
B^{\prime \prime}\left(t_{p}\right)=\min \left(\sqrt{4 \alpha D_{m} t_{p} / R_{m}} W\left(t_{p}\right)+\operatorname{sign}\left(q_{f m}\right) \max \left(\frac{\left|q_{f m}\right| t_{p}}{\theta_{m}}, 2 b\right), B\right)
$$

where $\alpha, D_{m}, R_{m}, \theta_{m}, q_{f m}$, and $2 b$ are the empirical coefficient, the effective matrix diffusion coefficient, the retardation factor in the matrix, the volumetric water content in the matrix, the water flux through the fracture-matrix interface and the effective fracture aperture, respectively. The weighting function $W\left(t_{p}\right)$ in $(2)$ was used to account for the secondary effects of the neighboring fractures on the expansion of the activity range. This enhanced particle-tracking method was shown, through comparison to analytical solutions developed by Sudicky and Frind (1982), to accurately simulate transport within the fracture-matrix system without fracture-matrix water flow. However, the above scheme had not yet been tested for a transport system with significant fracture-matrix water flow because no analytical solutions are available for such a system. Furthermore, the effect of the fracture-matrix water flow on the activity range was simply accounted for by assuming a constant fracture-matrix water velocity from the fracturematrix interface into the rock matrix (Pan and Bodvarsson, 2001), while the fracture-matrix water flow should decrease away from the fracture-matrix interface and cease at the center of the matrix block. Therefore, the objectives of this study are (1) to investigate the influence of the fracture-matrix water flow on the activity range and (2) to develop improved schemes for calculating the activity range that accounts for the spatially varied water velocity along the fracture-matrix dimension more accurately. The improved particle-tracking model will be verified against analytical solutions and a MINC model.

\section{Theory}

The purpose of calculating the activity range is not to describe the details of particle distribution within the matrix block. Instead, it is used to improve the accuracy of calculating the particle- 
transfer probability between fractures and matrix. Furthermore, in the dual-continuum model, the fracture-matrix mass transfer is treated as a local process (or subgrid process). In other words, the fracture-matrix connection actually constitutes the fourth dimension (i.e., the fracturematrix dimension) superimposed upon a regular 3-D space. Therefore, we can focus on developing a scheme that accounts for the effect of the fracture-matrix water flow on the expansion of particle activity range. Following the lead of Pan and Bodvarsson's (2001) approach, we can start with the 1-D governing equation (in the fracture-matrix dimension) of the advection-diffusion process in the matrix responding to a pulse injection in the fractures:

$$
\frac{\partial}{\partial \mathrm{t}_{\mathrm{p}}}\left[\mathrm{R}_{\mathrm{m}} \mathrm{C}\right]=\frac{\partial}{\partial \mathrm{s}}\left(\mathrm{D}_{\mathrm{m}} \frac{\partial \mathrm{C}}{\partial \mathrm{s}}\right)-\frac{\partial\left[q(s) C / \theta_{m}\right]}{\partial s}
$$

where $s$ is the distance from the fracture-matrix interface into the matrix. Note that $q(s)$ is the water flux along the fracture-matrix dimension, decreasing with s (e.g., $\mathrm{q}(0)=\mathrm{q}_{\mathrm{fm}}$ and $\mathrm{q}(\mathrm{B})=0$ ). Although the detailed spatial distribution $\mathrm{q}(\mathrm{s})$ is not available in the flow-field information provided by the dual-continuum flow simulator, a linear distribution is a more reasonable approximation than a constant assumption. That is:

$$
q(s)=q_{f m}\left(1-\frac{s}{B}\right) \quad 0 \leq s \leq B
$$

By inserting (4) into (3) and expanding the second term on the right hand of (3), we can rewrite (3) as follows:

$$
\frac{\partial}{\partial \mathrm{t}_{\mathrm{p}}}\left[\mathrm{R}_{\mathrm{m}} \mathrm{C}\right]=\frac{\partial}{\partial \mathrm{s}}\left(\mathrm{D}_{\mathrm{m}} \frac{\partial \mathrm{C}}{\partial \mathrm{s}}\right)-\frac{q}{\theta_{m}} \frac{\partial C}{\partial s}+\frac{q_{f m}}{\theta_{m} B} C
$$

We can further approximate $\mathrm{q}$ in the second term on the right-hand side of (5) with the average value of $q$ over $[0, B]$ (i.e., $0.5 \mathrm{q}_{\mathrm{fm}}$ ) to facilitate an analytical solution as follows(Bear 1972): 


$$
\mathrm{C}\left(\mathrm{s}, \mathrm{t}_{\mathrm{p}}\right) \approx \frac{2 \mathrm{M}_{\mathrm{s}}}{\left(4 \pi \mathrm{D}_{\mathrm{m}} \mathrm{t}_{\mathrm{p}} / \mathrm{R}_{\mathrm{m}}\right)^{1 / 2}} \exp \left[-\frac{\left(\mathrm{s}-0.5 \mathrm{q}_{\mathrm{fm}} \mathrm{t}_{\mathrm{p}} / \theta_{\mathrm{m}}\right)^{2}}{4 \mathrm{D}_{\mathrm{m}} \mathrm{t}_{\mathrm{p}} / \mathrm{R}_{\mathrm{m}}}+\frac{q_{f m} t_{p}}{\theta_{m} B}\right]
$$

Similar to the way used by Pan and Bodvarsson (2001) for deriving the $B^{*}\left(t_{p}\right)$ relationship from the pure diffusion solution, we can solve the basic relationship between the activity range $\mathrm{B}^{*}$ and the particle's age from the following equation:

$$
\frac{\left(\mathrm{B}^{*}-0.5 \mathrm{q}_{\mathrm{fm}} \mathrm{t}_{\mathrm{p}} / \theta_{\mathrm{m}}\right)^{2}}{4 \mathrm{D}_{\mathrm{m}} \mathrm{t}_{\mathrm{p}} / \mathrm{R}_{\mathrm{m}}}-\frac{q_{\mathrm{fm}} t_{p}}{\theta_{m} B}=\alpha
$$

The constant $\alpha$ is the same as used in (2). Finally, the new schemes for calculating the activity range can be written as follows:

$$
B^{\prime \prime}\left(t_{p}\right)=\min \left(\sqrt{\left(\alpha+\frac{q_{f m} t_{p}}{\theta_{m} B}\right) 4 D_{m} t_{p} / R_{m}} W\left(t_{p}\right)+\frac{q_{f m} t_{p}}{2 \theta_{m}}, B\right)
$$

The weighting function $\mathrm{W}\left(\mathrm{t}_{\mathrm{p}}\right)$ is the same as used in (2) to account for the secondary effects of the neighboring fractures on the expansion of the activity range in a parallel fracture system.

The new scheme (8) can be incorporated into the existing dual-continuum particle tracking code (DCPT) simply by replacing (2) with (8) in calculating the fracture-matrix particle transfer probability.

\section{Results and Discussions}

\subsection{Test Case 1}


This test case is the same as the larger fracture spacing $(10 \mathrm{~m})$ case used by Pan and Bodvarsson (2001). An analytical solution for solute transport in such fractured porous media was derived by Sudicky and Frind (1982). This solution is based on the assumptions that (1) the solute transport between fractures and the matrix occurs through matrix diffusion in the horizontal direction only, and that (2) matrix advection and diffusion in the vertical direction can be ignored. In addition, the initial solute concentration is zero in the system, and the concentration at inlets of fractures is constant for time $t>0$. Table 1 shows the relevant parameters for the test case. Although this test case cannot be used to verify whether or not the new improvement is successful in modeling the fracture-matrix mass transfer under the condition that fracture-matrix water flow exists, the analytical solution provides a good tool by which to verify the accuracy of the new numerical method and the overall implementation of the particle-tracking method. Furthermore, the newly improved scheme should also work for the cases in which the previous scheme works.

Table 1. Parameters Used for the Transport Problem in a Parallel Fracture System

\begin{tabular}{|l|l|}
\hline \multicolumn{1}{|c|}{ Parameter } & \multicolumn{1}{c|}{ Value } \\
\hline Molecular diffusion coefficient $\left(D_{\mathrm{m}}\right)$ & $2.5 \times 10^{-11} \mathrm{~m}^{2} / \mathrm{s}$ \\
\hline Fracture spacing (2B) & $10.0 \mathrm{~m}$ \\
\hline Fracture aperture (2b) & $2 \times 10^{-5} \mathrm{~m}$ \\
\hline Retardation factor $(R)$ & 30 \\
\hline Volumetric Water content in matrix & $0.1 \mathrm{~m}^{3} / \mathrm{m}^{3}$ \\
\hline Velocity in fracture & $1.1574 \times 10^{-5} \mathrm{~m} / \mathrm{s}$ \\
\hline Grid spacing & $0.5 \mathrm{~m}$ \\
\hline Matrix volume per cell & $2.5 \mathrm{~m}^{3}$ \\
\hline Fracture volume per cell & $0.5 \times 10^{-5} \mathrm{~m}^{3}$ \\
\hline Fracture-matrix interface area & $0.5 \mathrm{~m}^{2}$ \\
\hline $\begin{array}{l}\text { Distance from the source of tracer to the } \\
\text { observation point }\end{array}$ & $36.5 \mathrm{~m}$ \\
\hline
\end{tabular}




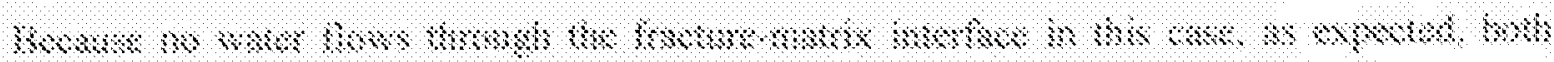

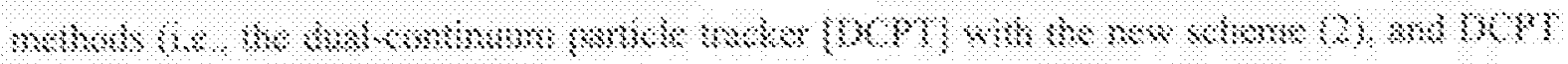
Wh monos

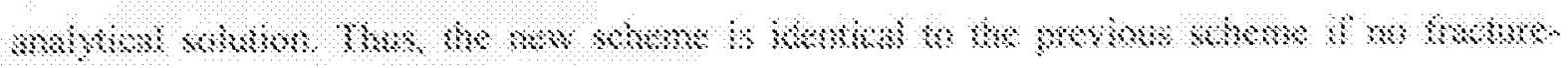

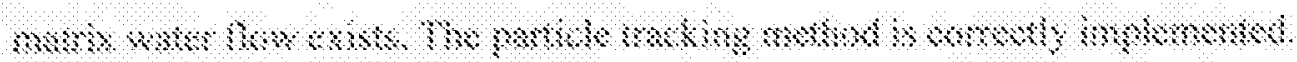

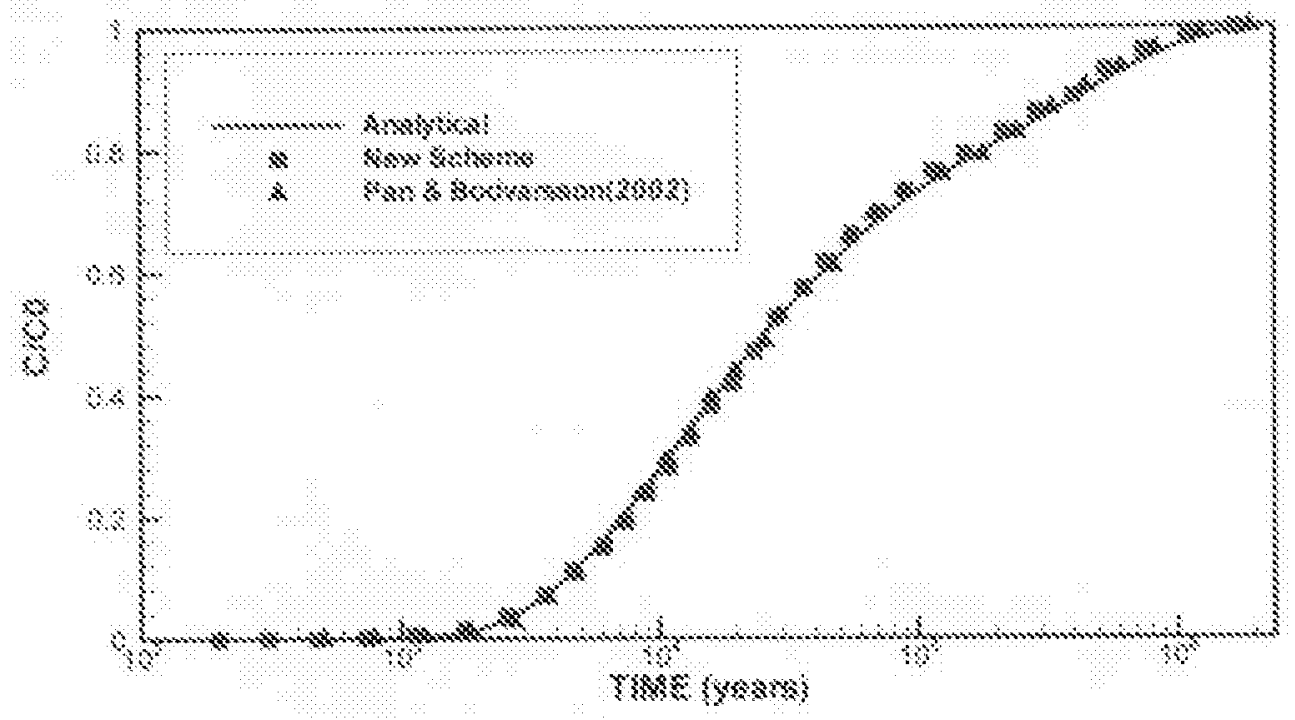

\$

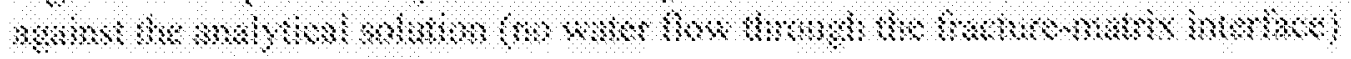

\section{T० $\mathrm{Sm}$}

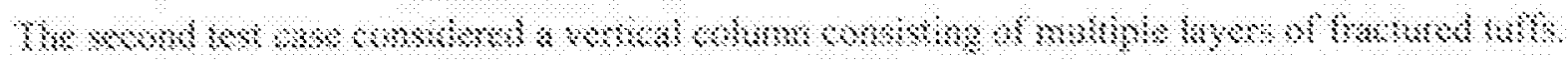
$\alpha$ का

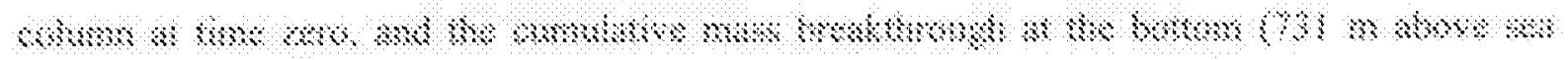

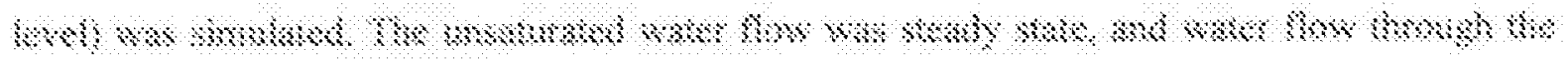

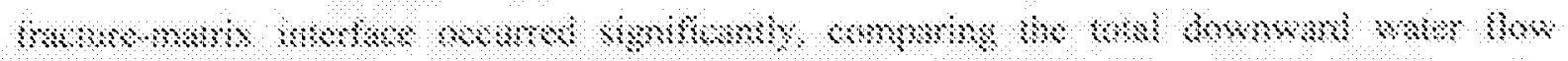


S

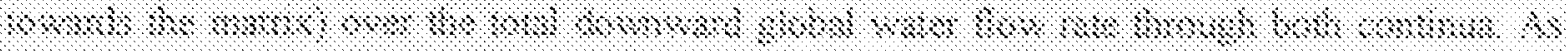

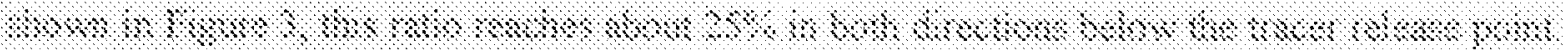

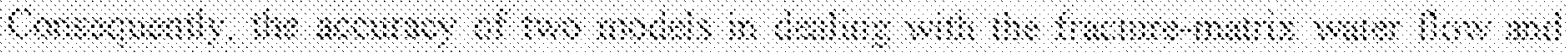

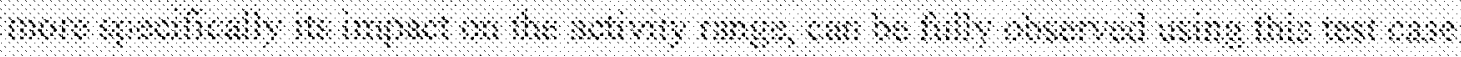

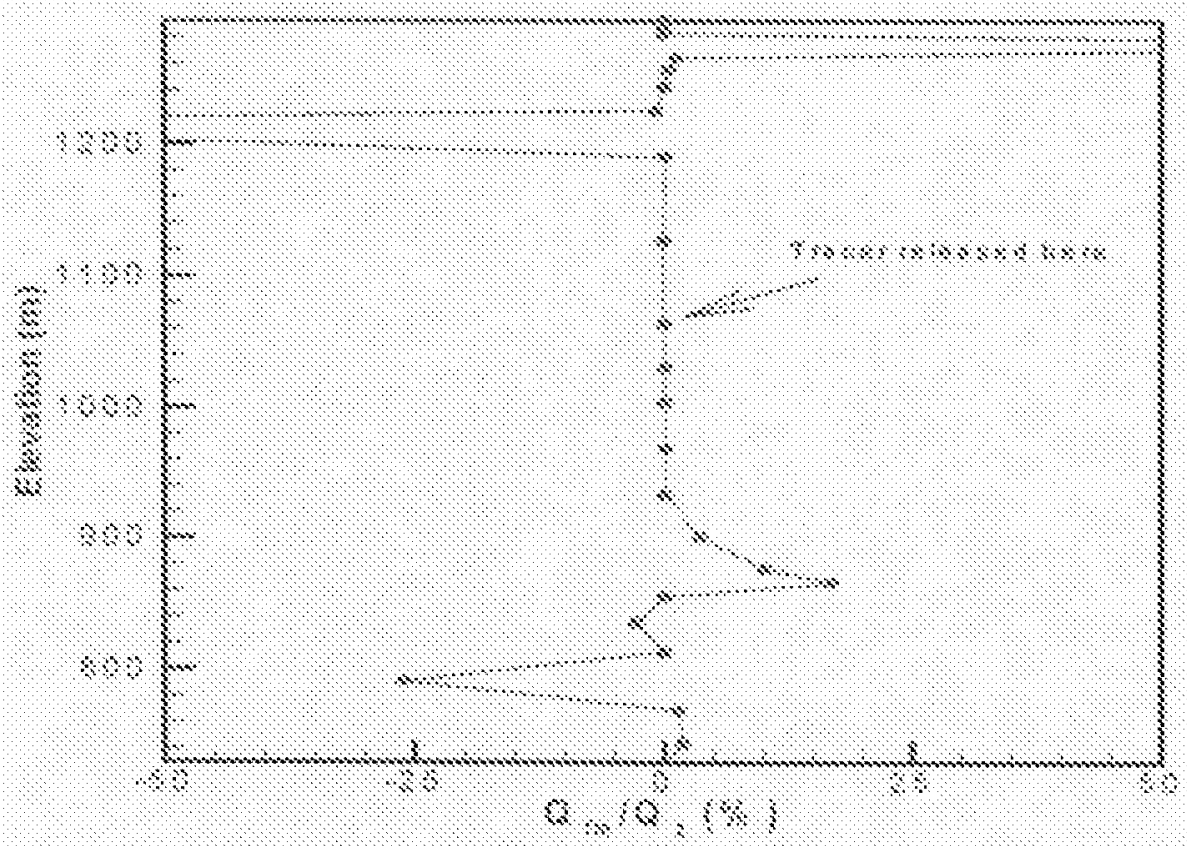

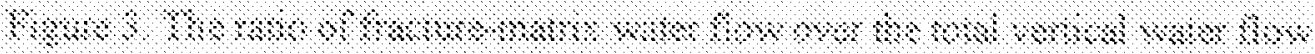

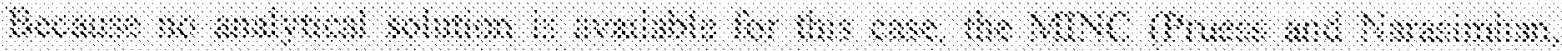

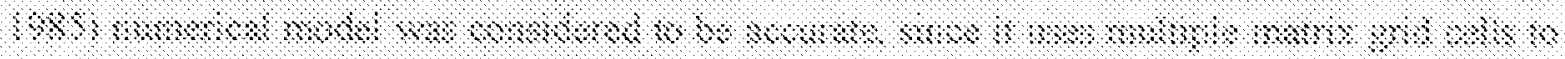

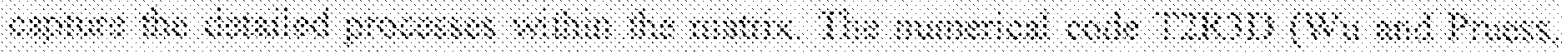

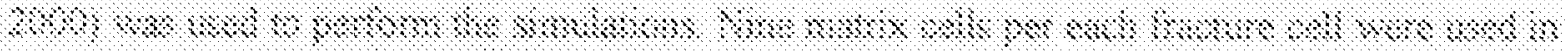


the MINC model. A dual-permeability (2k) model (one matrix cell per each fracture cell) was also included as a reference.

As shown in Figure 4, while it effectively solves the early breakthrough problem associated with the conventional dual-continuum model (e.g., T2R3D-2k), the particle tracker with the previous scheme (Equation 2) does not compare well with the MINC model (e.g., T2R3D-MINC), especially at later times. It predicts a much more smeared breakthrough curve than that predicted by the more accurate MINC model. On the other hand, the particle tracker with the new improved scheme (Equation. 8) predicts breakthrough curves that are almost identical to the MINC model (Figure 4). In other words, the particle tracker with the new scheme can attain accuracy similar to the MINC model in predicting the breakthrough curves, without using MINC's additional matrix grid cells ( 9 in this case), provided that the flow fields are the same.

In terms of physical mechanism, the activity range of particles is directly related to the peneration depth into the matrix after particles are injected into the fractures. When fracturematrix water flow does not occur, the expanding of the penetration depth is purely a result of matrix diffusion. Consequently, the penetration depth can be described as an expanding process proportional to the square root of time (i.e., the particle's age). However, when fracture-matrix water flow is significant compared to the total global water flow, the square root rule can no longer apply because of the interaction between matrix diffusion and fracture-matrix water flow. The effects of fracture-matrix water flow on the expansion of the activity range or penetration depth are not only reflected in direct advection movements but also in enhancing (when the fracture-matrix flow is towards the matrix) or reducing (when the fracture-matrix flow is towards the fractures) the matrix difussion process. The success of the improved scheme (8) reveals that 


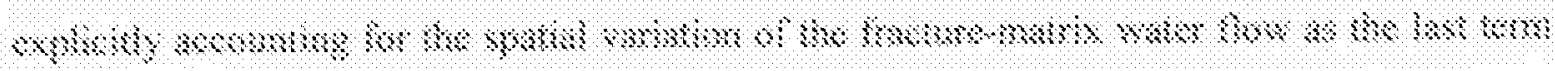

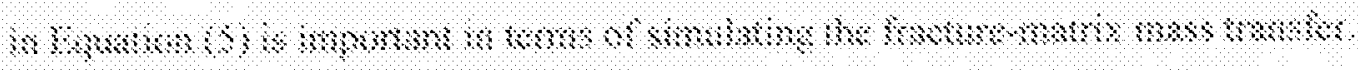

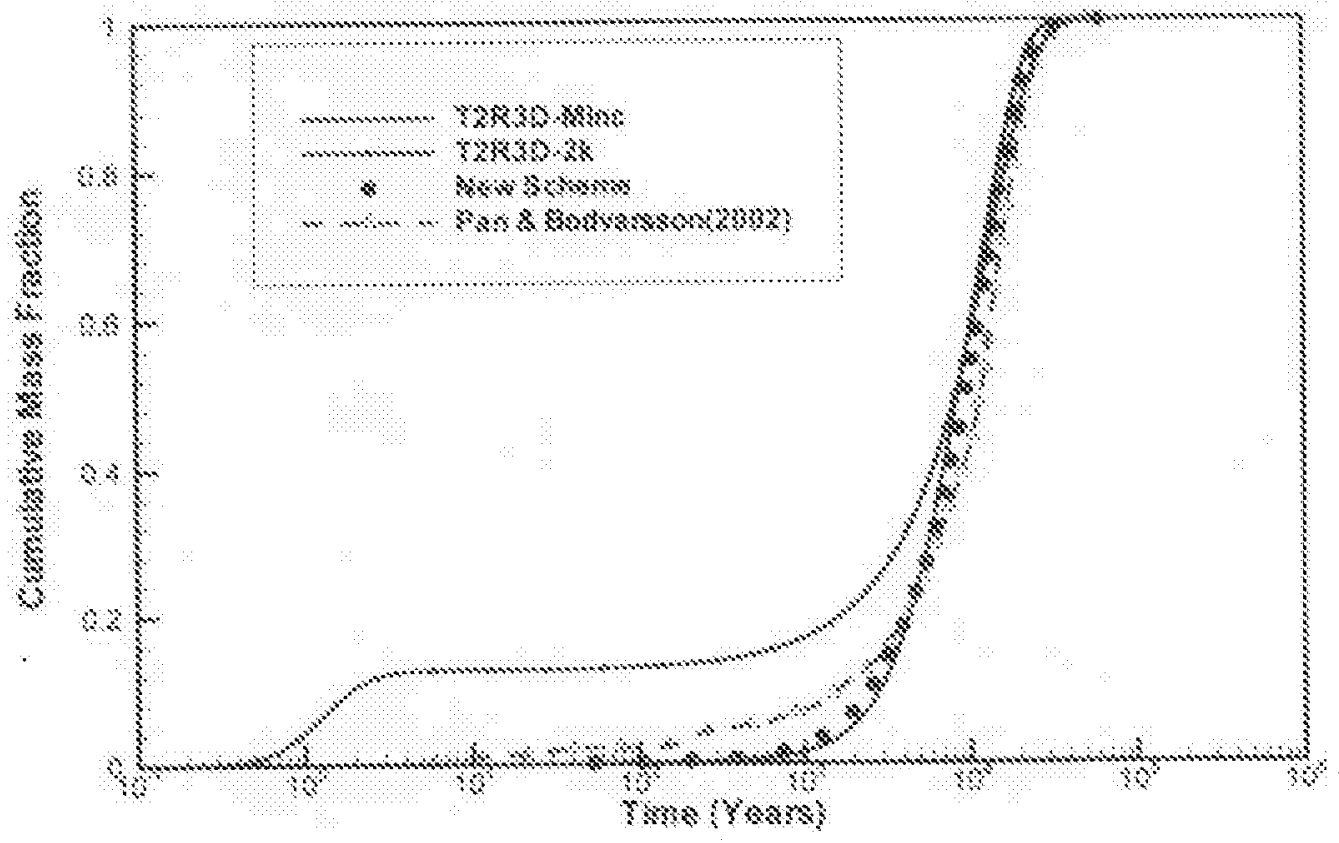

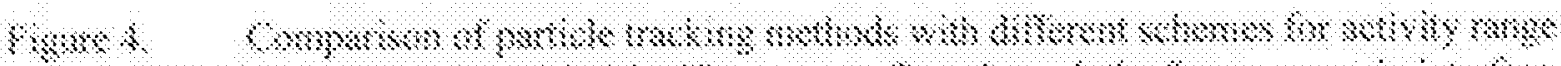

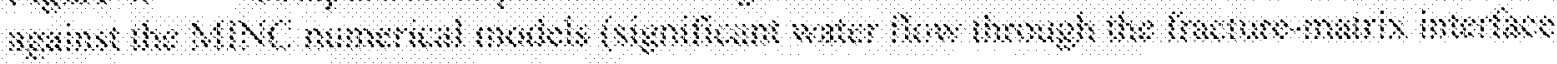
$\leftrightarrow \mathrm{s} \times \mathrm{s}$

\section{4. $5 \mathrm{mos} \times \mathrm{man} 6 \mathrm{~m}$}

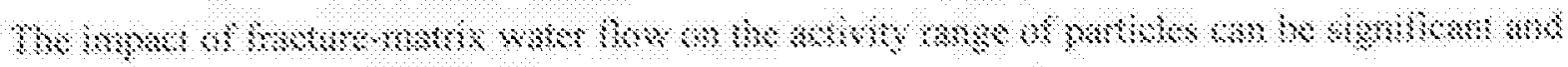

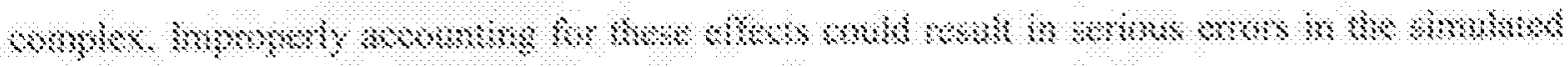

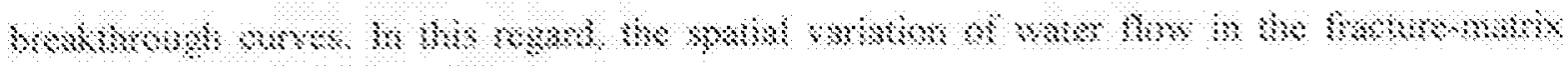

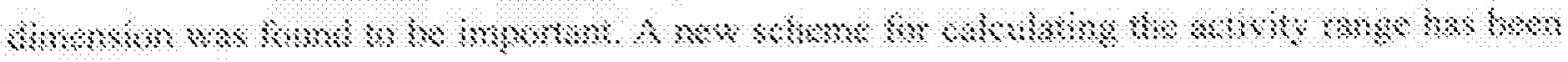


proposed, based on an analytical solution of the simplified advection-dispersion equation along the fracture-matrix dimension. The modified dual-continuum particle tracker (DCPT) with this new scheme was found to be as accurate as a multiple-continuum numerical model (MNNC). While the MINC model solves transient penetration depth problems by using more matrix cells (e.g., 9 matrix cells in the case presented in this note), the modified dual-continuum particle tracker does not need any additional matrix cells (besides the one matrix cell). The resulting advantages in efficiency are obvious, especially for large-scale transport modeling.

\section{Acknowledgments}

The authors would like to thank Guoping Lu and Dan Hawkes for their internal review and thoughtful comments. This work was supported in part by the Director, Office of Civilian Radioactive Waste Management, U.S. Department of Energy, through Memorandum Purchase Order QA-B004220RB3X between Bechtel SAIC Company, LLC and the Ernest Orlando Lawrence Berkeley National Laboratory (Berkeley Lab). The support is provided to Berkeley Lab through the U.S. Department of Energy Contract No. DE-AC03-76SF00098.

\section{References}

Pan, L., H.H. Liu, M. Cushey, and G. S. Bodvarsson, DCPT V1.0 - New particle tracker for modeling transport in dual continua media, Users' manual. Report LBNL-42958, Lawrence Berkeley National Laboratory, Berkeley, CA, 2001.

Pan, Lehua, and Gudmundur S. Bodvarsson, Modeling transport in fractured porous media with the random-walk particle method: The transient activity range and the particle transfer probability. Water Resources Research, 38(6):1029-1037, 2002. 
Pruess, K., and T. N. Narasimhan, A practical method for modeling fluid and heat flow in fractured porous media. Soc. Pet. Eng. J. 25:14-26, 1985.

Sudicky, E. A., and E. O. Frind, Contaminant transport in fractured porous media: Analytical solutions for a system of parallel fractures, Water Resources Research, 18:1634-1642, 1982.

Wu, Y. S., and K. Pruess, Numerical simulation of non-isothermal multiphase tracer transport in heterogeneous fractured porous media. Advances in Water Resources. 23:669-723, 2000. 